\title{
Target validation by array
}

In this month's issue of Nature Medicine (4:1293-1301, 1998), scientists at Rosetta Inpharmatics (Kirkland, WA) and Stanford University School of Medicine Howard Hughes Medical Institute, California present an elegant way of streamlining the validation of primary and secondary drug targets. They use cDNA microarrays to compare genome-wide gene expression patterns in wild-type, drugtreated yeast with those in a series of yeast gene deletion mutants. Reasoning that either deletion of a gene or pharmacological inhibition of its protein would result in the same expression

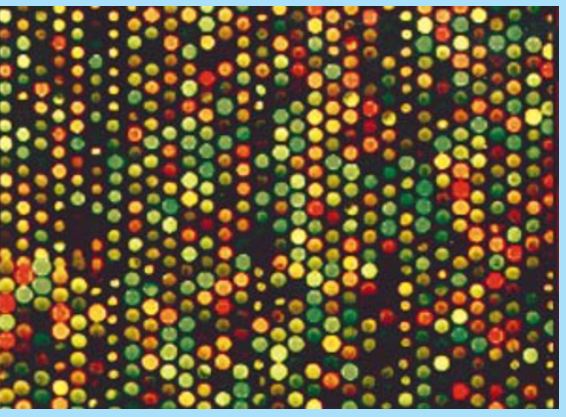

Yeast genome expression array showing the effects of 3-aminotriazole. profile, the authors confirmed that calcineurin - a phosphatase implicated in a number of calcium-dependent pathways-is the primary target of the immunosuppressants FK506 and cyclosporine A. The investigators took the additional step of determining the expression profile in the drug-exposed mutant yeast strain to verify the primary target. This "decoder" step also allowed secondary pathways that are affected by the drug independently of the primary target to be identified. FK506 was found to affect at least two additional pathways that were independent of its effects on calcineurin. The authors propose that their approach "may help to identify compounds with undesirable secondary drug target effects that only come to light late in the drug discovery process."

\section{Tunable gene regulation}

Using short RNA sequences called aptamers, which bind to a small molecule compound with high affinity, researchers have developed a general approach for regulating gene expression at the level of translation. The technique, described in a recent issue of Science (282:296-298, 1998), entails inserting the complementary DNA for an aptamer into the untranslated region of a gene. The small molecule-a dye that can diffuse easily through cell membranes - can then bind specifically to the aptamer-containing transcripts and prevent ribosomes from translating them. In the absence of the dye, translation of the gene can proceed normally. Michael Green, a researcher in the Howard Hughes Medical Institute at the University of Masachusetts Medical Center (Worcester, MA) and the senior author on the study, explains that "this provides a completely independent way of regulating gene expression. It's not just an onoff switch, but you can actually modulate the activity by adjusting the concentration [of the dye]." This flexibility would be useful for studies of gene regulation pathways, and while the approach has only been demonstrated in tissue culture, the team is also hoping to develop it into an inducible gene knock-out system for laboratory animals.

Research News Briefs written by Alex Castellino, Alan Dove, LeeAnn Leshko, and Orla Smith.

\section{Quelling xenoreactive Abs}

Human antibody responses to foreign organs continue to thwart realization of current xenotransplantation approaches. A report in Science (281:1845-1847, 1998) suggests one potential solution may be to tolerize human $\mathrm{B}$ cells by autologous transplant using bone marrow cells displaying the pig hyperacute rejection epitope $\alpha \mathrm{Gal}$. After showing that Vero cells transduced with $\alpha(1-3)$ galactosyltransferase $(\alpha \mathrm{GT})$ produce the porcine $\alpha \mathrm{Gal}$ epitope, John Iacomini and colleagues from Massachusetts General Hospital and the Harvard University Medical School (Cambridge, MA) reconstituted lethally irradiated $\alpha$ GT knockout mice (which normally produce $\alpha \mathrm{Gal}$ reactive antibodies) with autologous bone marrow cells transduced with or without $\alpha$ GT. Several weeks after reconstitution, sera from control animals reconstituted with syngeneic bone marrow lacking $\alpha \mathrm{GT}$ showed stable levels of xenoreactive antibodies, whereas sera from animals reconstituted with $\alpha$ GT-transduced cells showed no xenoreactive antibodies, as detected by ELISA, flow cytometry, and the more sensitive fluorescence-based complement-mediated cytotoxicity assay. Iacomini feels that "being able to reprogram the host immune system via the route of molecular chimerism will have important bearings in the fields of transplantation biology and autoimmune disease." The group is currently testing this approach in a baboon model for xenotransplantation.

\section{Self-help chlamydial vaccine}

Scientists from the National Institute of Allergy and Infectious Disease/National Institutes of Health at the Rocky Mountain Laboratory (Hamilton, MO) have generated the first effective vaccine against chlamydial genital infections. By introducing ex vivo sensitized dendritic cells (DCs) into naive mice, they successfully induced T-cell immunity in vivo against the elusive pathogen, Chlamydia trachomatis. In a recent issue of The Journal of Experimental Medicine (188:809-819, 1998), Harlan Caldwell and colleagues show that chlamydia-pulsed DCs phagocytose heat-killed bacteria, correctly present chlamydial antigens, and induce CD4 T-cell cytokine responses similar to those of chlamydia-infected mice. More importantly, the approach resulted in protective immunity against chlamydial genital tract infection in mice equal to immunity obtained after infection with live bacteria. "This study shows it's feasible to generate an efficacious vaccine against chlamydial genital tract infection," claims Caldwell. "It could be very useful for developing vaccines against other infectious diseases that warrant aggressive, unconventional therapies."

\section{Quantum leap for labeling}

A joint research team from Lawrence Berkeley National Laboratory and the University of California at Berkeley and a separate research team from Indiana University, Bloomington, have independently used quantum dots (QD) - light-emitting semiconductor nanocrystals-as fluorescent markers in biological systems. Both teams chemically altered the surfaces of these nanocrystals to make them water soluble and biocompatible and linked them to tracer molecules that would follow target proteins in cells. The Berkeley team led by Shimon Weiss and Paul Alivisatos used two different size nanocrystals to simultaneously label actin filaments and nuclei in fixed fibroblasts, demonstrating that QD probes can be used in tandem. The Indiana group led by Shuming Nie covalently linked QD directly to proteins and showed that these bioconjugates can be incorporated into living cells and can recognize target immunomolecules in immunoassays. Nie claims that "quantum dots are the obvious choice for developing a new class of labels since they are brighter and more stable than conventional dyes, and their emission colors can be fine-tuned by changing the size of the dot." Alivisatos adds, "the main advantage of the semiconductor nanocrystals will be the ability to use several labels simultaneously using one laser to excite all probes." The findings are reported in a recent issue of Science (281:2013-2016; 2016-2018, 1998). 